\title{
Utility of Fluorodeoxyglucose Positron Emission Tomography/Computed Tomography in Patients with Fever of Unknown Origin Diagnosed as Lymphoma
}

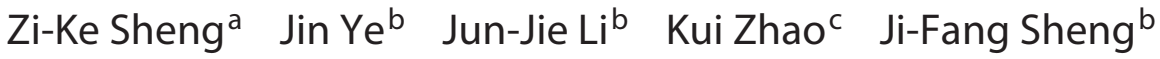 \\ anstitute of Antibiotics, Huashan Hospital, Fudan University, Shanghai, and bepartment of Infectious DIseases, \\ State Key Laboratory for Diagnosis and Treatment of Infectious Diseases, and 'PET Center, First Affiliated Hospital, \\ School of Medicine, Zhejiang University, Hangzhou, PR China
}

\section{Key Words}

Fever of unknown origin - Positron emission tomography . Computed tomography · Diagnosis · Lymphoma

\section{Abstract \\ Objective: To assess the efficacy of fluorine-18 fluorodeoxy- glucose positron emission tomography $\left({ }^{18} \mathrm{~F}-\mathrm{FDG} \mathrm{PET}\right) / \mathrm{com}$ - puted tomography (CT) in the diagnosis of patients with fe- ver of unknown origin (FUO), who were finally diagnosed as lymphoma. Subjects and Methods: A retrospective study was performed in the First Affiliated Hospital, School of Med- icine of Zhejiang University, China, from March 2009 to March 2012. The PET/CT images of consecutive patients with FUO were analyzed. Within 1 week of PET/CT scanning, additional histological tests were also performed if clinically needed. Results: A total of 73 consecutive patients were included. Of these, 34 (47\%) had a PET/CT finding suggestive of the pres- ence of lymphoma and 29 (85\%) had a diagnosis of confirmed lymphoma; 39 (53\%) had a PET/CT result revealing the ab- sence of lymphoma and 4 (10\%) were diagnosed by biopsy as having lymphoma, . The most frequent lymphoma diagno-}

sis was peripheral T cell lymphoma $(n=16 ; 55 \%)$, followed by diffuse large $B$ cell lymphoma $(n=9 ; 31 \%)$. The accuracy of PET/CT was $88 \%$. Conclusion: In this study, PET/CT had high diagnostic accuracy in patients with FUO resulting from lymphoma, which indicated that PET/CT scanning was a valuable diagnostic tool for these groups of patients with FUO.

c) 2014 S. Karger AG, Basel

\section{Introduction}

In the past decades, neoplastic causes have exceeded infectious causes as the most common reasons for fever of unknown origin (FUO) $[1,2]$. Lymphoma is one type of neoplasm that is classically associated with FUO, and has become the main cause for FUO in adults [3]. Timely diagnosis of lymphoma, especially in patients with FUO, may affect both treatment and prognosis. Confirmation of a diagnosis of lymphoma mainly depends on histology, such as biopsy and surgery. However, these procedures are invasive and usually a limited part of tissue or organ is involved.

\begin{tabular}{ll}
\hline KARGER & $\begin{array}{l}\text { ๑ 2014 S. Karger AG, Basel } \\
1011-7571 / 14 / 0235-0437 \$ 39.50 / 0 \quad \text { Karger }\end{array}$ \\
E-Mail karger@karger.com & $\begin{array}{l}\text { Thisis an Open Access article licensed under the terms of the } \\
\text { Creative Commons Attribution-NonCommercial 3.0 Un- } \\
\text { ported license (CC BY-NC) (www.karger.com/OA-license), } \\
\text { applicable to the online version of the article only. Distribu- } \\
\text { tion permitted for non-commercial purposes only. }\end{array}$
\end{tabular}

Dr. Ji-Fang Sheng

State Key Laboratory for Diagnosis and Treatment of Infectious Diseases

First Affiliated Hospital, School of Medicine, Zhejiang University

79 Qingchun Road, Hangzhou 310003 (PR China)

E-Mail jifangsheng9200@163.com 
Fluorine-18 fluorodeoxyglucose positron emission tomography $\left({ }^{18} \mathrm{~F}-\mathrm{FDG} \mathrm{PET}\right) /$ computed tomography (CT) is noninvasive and scans a wide range of the body. In contrast to conventional imaging techniques, PET/CT can provide the useful metabolic and functional information of the foci through a whole-body scan. PET/CT has shown good performance regarding the management of patients with lymphoma $[4,5]$.

In patients with FUO, ${ }^{18} \mathrm{~F}-\mathrm{FDG} \mathrm{PET} / \mathrm{CT}$ and ${ }^{18} \mathrm{~F}-\mathrm{FDG}$ PET have the potential ability to diagnose lymphomas, as well as infections and noninfectious inflammatory diseases, which are the three main causes of FUO [6-9]. However, the diagnostic accuracy in these causes varies. To our knowledge, studies on the diagnostic value of $\mathrm{PET} / \mathrm{CT}$ in patients with FUO diagnosed as lymphoma are currently limited. Therefore, this study aimed to assess the diagnostic utility of PET/CT in patients with FUO resulting from lymphoma.

\section{Subjects and Methods}

\section{Patients}

This retrospective study was done at the First Affiliated Hospital (an approximately 2,500-bed hospital), School of Medicine of Zhejiang University, China. From March 2009 to March 2012, data of all consecutive patients with FUO who had PET/CT scanning were retrospectively analyzed. Included in the PET/CT scanning were patients with FUO to exclude hematologic malignancies (mainly lymphoma) at the initial stage of diagnosis. FUO was defined as an illness with recurrent fever of over $38.3^{\circ} \mathrm{C}$ lasting 3 weeks or more, and without a diagnosis after 1 week of detailed clinical investigation in this study [10]. Excluded patients were those who either did not have a classic FUO according to the definition mentioned above or who had any previous treatment for neoplasms, as well as patients with an immunocompromised condition. Pathologic diagnoses were grouped according to the World Health Organization (WHO) classification of lymphoma (2008) [11].

The PET/CT images of 133 patients were reviewed. Of these, 56 patients were excluded in whom PET/CT imaging was done to find the underlying nonmalignant lesions or potential foci that were difficult to detect using conventional imaging. The remaining 77 patients with suspicion of lymphoma who met the inclusion criteria were enrolled. The clinical data of 1 of these patients were not available, and 3 dropped out of follow-up. Thus, 73 patients (45 male, 28 female; mean age 52 years; range 18-78 years) were then included for further analysis. Of the 73 patients, 65 (89\%) were referred from a local or community hospital because of FUO without a final diagnosis.

\section{Diagnostic Work-Up}

Detailed physical examination, assessment of medical history and routine laboratory tests were conducted for all the patients. $\mathrm{PET} / \mathrm{CT}$ scanning was done at the time of initial diagnosis by phy- sicians. A PET/CT-guided biopsy (bone marrow or peripheral lymph nodes) or surgery (such as splenectomy) was performed within 1 week of PET/CT scanning. A follow-up of more than 1 year was done for all the enrolled patients. Final diagnosis was established based on the pathological findings, clinical course and criteria, microbiology, and follow-up.

\section{${ }^{18}$ F-FDG PET/CT}

After a fast of at least $6 \mathrm{~h}$, patients received an intravenous ${ }^{18} \mathrm{FDG}$ administration of $5.5-7.4 \mathrm{MBq} / \mathrm{kg}$ of body weight. Patients were hydrated with $1,000 \mathrm{ml}$ of water $1 \mathrm{~h}$ prior to image acquisition. Blood glucose levels were checked in all patients before ${ }^{18} \mathrm{FDG}$ injection to ensure that they all had a blood glucose level greater than $160 \mathrm{mg} / \mathrm{dl}$. Whole-body PET/CT scans were acquired using a combined PET/CT scanner (Siemens Biograph Sensation 16; Siemens, Berlin, Germany). Whole-body CT and PET from the meatus of the ear to the mid-thigh was performed approximately $1 \mathrm{~h}$ after FDG injection. The procedure for data acquisition was as follows: 16-section multidetection row CT scanning was performed first, from the head to the mid-thigh with $120 \mathrm{kV}, 100 \mathrm{~mA} \mathrm{~s}$. A tube rotation time of $0.5 \mathrm{~s}$ and a $5-\mathrm{mm}$ section thickness were matched to the PET section thickness. Immediately after the CT scan, a whole-body emission PET scan was obtained with 3-min acquisition per bed position using a 3-dimensional acquisition mode. Attenuation-corrected PET images were reconstructed with an ordered-subset expectation maximization iterative reconstruction algorithm ( 8 subsets, 3 iterations). $\mathrm{PET}, \mathrm{CT}$ and fused PET/CT images were generated and reviewed on a computer workstation (Virtual Place version 3.0035; AZE, Tokyo, Japan). The PET/CT scans were independently interpreted by two staff members of the PET center. The increased uptake of ${ }^{18} \mathrm{~F}$-FDG with an intensity higher than that of surrounding tissues in at least one area was considered as positive, while no sites of increased ${ }^{18} \mathrm{~F}$-FDG uptake were defined as negative. Disagreements between respective independent interpretations were resolved by consensus. All 73 patients had a single PET/CT scan.

\section{Clinical Evaluation of PET/CT}

A positive $\mathrm{PET} / \mathrm{CT}$ finding was defined as a focal or multifocal abnormal FDG uptake in tissue or bone marrow, suggestive of the presence of lymphoma according to the standardized uptake value; a negative PET/CT finding was a PET/CT image without an elevated FDG uptake in tissue and/or bone marrow, suggesting the absence of lymphoma. A positive PET/CT result was considered a true positive when the diagnosis of lymphoma was confirmed by biopsy or surgery, and was regarded as a false positive when biopsy, surgery or follow-up revealed the absence of lymphoma. A negative PET/CT finding was considered a true negative when no lymphoma was diagnosed, despite additional histological tests and a follow-up period of more than 1 year. A false negative was defined when lymphoma was verified by additional pathological procedures or follow-up.

\section{Statistical Analysis}

The 95\% confidence intervals (95\% CI) were calculated with the EBM calculator (www.cebm.utoronto.ca) for sensitivity, specificity, positive likelihood ratio (+LR), negative likelihood ratio $(-\mathrm{LR})$, positive predictive value and negative predictive value. 
Table 1. PET/CT and histological results of 33 patients with lymphomas

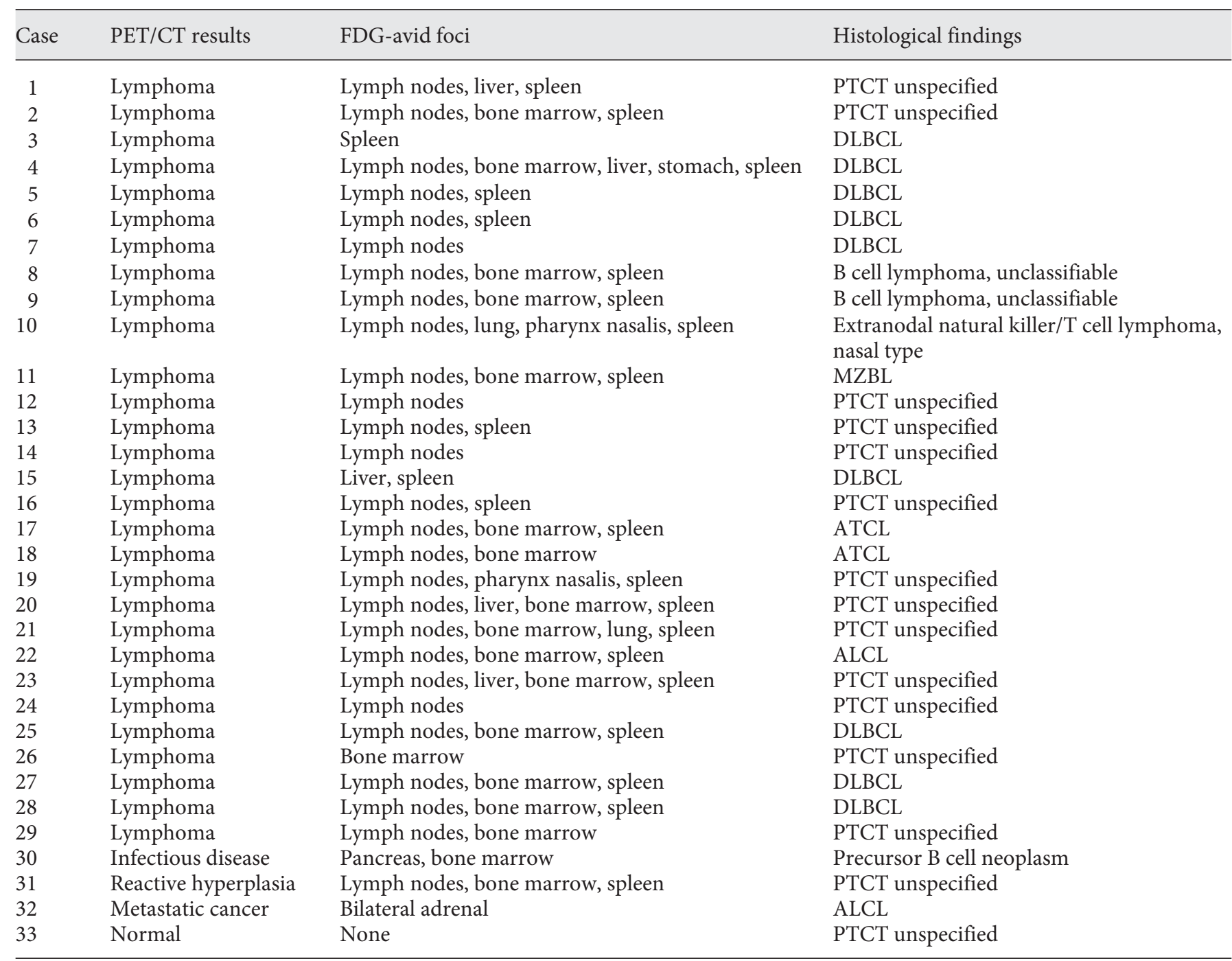

\section{Results}

\section{Patients}

Of the 73 patients, 33 (45\%) had a histological confirmation of lymphoma, while 40 (55\%) were without a diagnosis of lymphoma. Of the 33 patients with confirmed lymphoma, 4 had splenectomy, 14 had unilateral iliac crest biopsy with aspirate (BMB), and 15 had direct biopsy of the FDG-avid lesion (14 peripheral lymph nodes, 1 adrenal). The histological subtypes (table 1), according to the WHO classification, were diffuse large B cell lymphoma (DLBCL; $\mathrm{n}=9$ ), B cell lymphoma, unclassifiable $(n=2)$, precursor $B$ cell neoplasm $(n=1)$, marginal zone $\mathrm{B}$ cell lymphoma (MZBL; $\mathrm{n}=1$ ) and peripheral T cell lym- phoma (PTCL; total types, $\mathrm{n}=20$ ). The latter were comprised of PTCL, unspecified (PTCT unspecified; $\mathrm{n}=15$ ), angioimmunoblastic T cell lymphoma (ATCL; $n=2)$, extranodal natural killer/T cell lymphoma, nasal type ( $\mathrm{n}=$ 1) and anaplastic large cell lymphoma (ALCL; $n=2)$. Of the 40 patients without lymphoma, there were 77 pathological examinations, including $61 \mathrm{BMB}, 16$ nonbone biopsies, 1 splenectomy and 1 thoracotomy. The final diagnosis was established mainly based on clinical course and criteria, biopsies and follow-ups (table 2).

\section{${ }^{18} \mathrm{~F}-\mathrm{FDG}$ PET/CT}

Of the $73 \mathrm{PET} / \mathrm{CT}, 34$ (47\%) were positive while 39 (53\%) were negative. In the 33 patients with confirmed 
Table 2. Diagnoses and measures in 40 patients without lymphomas

\begin{tabular}{|c|c|c|c|c|c|c|c|}
\hline \multirow[t]{2}{*}{ Diagnosis } & \multirow[t]{2}{*}{ Total } & \multicolumn{2}{|c|}{$\mathrm{PET} / \mathrm{CT}$ results } & \multicolumn{4}{|c|}{ Measures } \\
\hline & & negative & positive & $\mathrm{CCC}$ & $\mathrm{CV}$ & $\mathrm{BO}$ & $\mathrm{FU}$ \\
\hline \multicolumn{8}{|l|}{ Infection } \\
\hline Tuberculosis infection & 4 & 4 & 0 & 4 & 0 & 0 & 0 \\
\hline Bacteremia & 3 & 3 & 0 & 2 & 1 & 0 & 0 \\
\hline Viral infection & 3 & 3 & 0 & 3 & 0 & 0 & 0 \\
\hline \multicolumn{8}{|l|}{ Solid tumor } \\
\hline Thyroid cancer & 1 & 0 & 1 & 0 & 0 & 1 & 0 \\
\hline Nasopharyngeal carcinoma & 1 & 1 & 0 & 0 & 0 & 1 & 0 \\
\hline Bone metastases & 3 & 3 & 0 & 0 & 0 & 3 & 0 \\
\hline \multicolumn{8}{|c|}{ Noninfectious inflammatory disease } \\
\hline Adult-onset Still's disease & 4 & 4 & 0 & 4 & 0 & 0 & 0 \\
\hline Sjögren's syndrome & 2 & 2 & 0 & 1 & 0 & 1 & 0 \\
\hline Hemophagocytic syndrome & 2 & 2 & 0 & 2 & 0 & 0 & 0 \\
\hline Necrotizing lymphadenitis & 1 & 1 & 0 & 0 & 0 & 1 & 0 \\
\hline Vasculitis & 1 & 1 & 0 & 0 & 0 & 1 & 0 \\
\hline Hematologic neoplasm & 1 & 1 & 0 & 0 & 0 & 1 & 0 \\
\hline Myelodysplastic syndrome & 1 & 1 & 0 & 0 & 0 & 1 & 0 \\
\hline No diagnosis & 14 & 10 & 4 & 0 & 0 & 0 & 14 \\
\hline Total & 40 & 35 & 5 & 16 & 1 & 9 & 14 \\
\hline
\end{tabular}

Values represent number of patients. $\mathrm{CCC}=$ Clinical course and criteria; $\mathrm{CV}=$ cultivation; $\mathrm{BO}=$ biopsy; $\mathrm{FU}=$ follow-up.

lymphoma, several sites of disease were FDG-avid detected by PET/CT (table 1). Increased FDG uptake in spleen and bone marrow was found in $23(70 \%)$ and 18 (55\%) patients with confirmed lymphoma, respectively.

\section{Clinical Evaluation of PET/CT and Histological Findings}

The PET/CT findings and the following biopsy/splenectomy results agreed in $64(88 \%, 64 / 73)$ patients, with concordant positive and concordant negative results in 29 and 35 patients, respectively. Thus, the 29 PET/CT scans were all considered to be beneficial in lymphoma diagnosis (true positive), and the 35 were called true negative.

Of the 33 patients with confirmed lymphoma, 29 (88\%) had a positive PET/CT finding, and only 4 were negative on PET/CT. Among the remaining 40 patients without a confirmation of lymphoma, 35 (88\%) had a negative PET/CT result and the absence of lymphoma was later established (true negative); 5 had a positive PET/ CT finding but lymphoma was not found (false positive). Of the 5 false positives, 1 patient had bone metastases confirmed by BMB, 1 had thyroid carcinoma established by a biopsy of the thyroid, and no lymphoma was found in the remaining 3, despite repeated biopsies and surgery. Therefore, the sensitivity, specificity, positive predictive value, negative predictive value and $+/-\mathrm{LR}$ of PET/CT in the diagnosis of lymphoma presenting as FUO were $88 \%$ (95\% CI 72-97\%), 88\% (95\% CI 73-96\%), 85\% (95\% CI 67-95\%), 90\% (95\% CI 76-97\%) and 7.03/0.14, respectively.

Among the 33 confirmed lymphoma patients, there were $4(12 \%, 4 / 33)$ whose PET/CT findings were not in concordance with histological results (table 1). In 1 patient the PET/CT scan showed a normal FDG uptake, but the bone marrow biopsy at iliac crest confirmed the presence of $\mathrm{T}$ cell lymphoma. The remaining $3 \mathrm{PET} / \mathrm{CT}$ scans suggested a diagnosis of infectious disease, reactive hyperplasia and metastatic cancer, respectively, but the biopsy results confirmed the diagnosis of lymphoma for each.

In this study, the PET/CT scan correctly diagnosed B cell lymphomas in $92 \%(12 / 13)$ of the patients with FUO, and DLBCL in $100 \%$ (9/9). In contrast, the PET/CT scan diagnosed PTCL (all types) in 85\% (17/20) of patients with FUO (fig. 1). Moreover, PET/CT also correctly diagnosed B cell lymphoma, unclassifiable $(\mathrm{n}=2)$, MZBL ( $\mathrm{n}$ $=1)$, ATCL $(\mathrm{n}=2)$, ALCL $(\mathrm{n}=1)$ and extranodal natural 

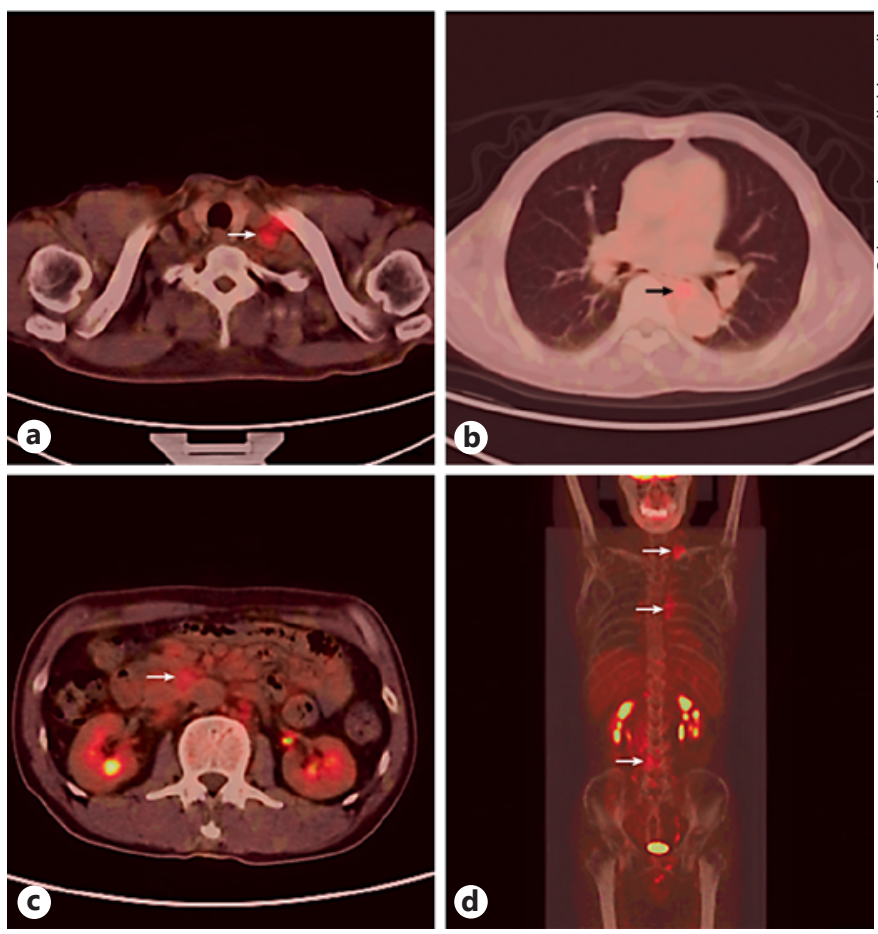

Fig. 1. A 64-year-old man who presented with FUO and underwent PET/CT for initial diagnosis. PET/CT revealed elevated FDG uptake in lymph nodes of the supraclavicular region with a maximum standardized uptake value (SUV max) of 3.50 (arrows; a, d), before the thoracic aorta with an SUV max of 3.45 (arrows; b, d), and in the abdomen with an SUV max of 3.80 (arrows; c, d). Lymph node biopsy was performed in the supraclavicular region lymph nodes, and the result revealed $\mathrm{T}$ cell lymphoma.

killer/T cell lymphoma, nasal type $(\mathrm{n}=1)$. However, the number of patients was too small to evaluate the role of $\mathrm{PET} / \mathrm{CT}$ scanning in these diseases.

\section{Discussion}

Our findings suggest that PET/CT is a valuable diagnostic procedure in FUO patients with lymphoma. In addition, the combination of PET/CT and additional PET/ CT-oriented histological tests showed a good performance in the diagnosis of lymphoma, as well as in ruling it out (negative predictive value 90\%; -LR 0.14). While the former was able to find the extent of diseases and localize the site of FDG-avid lesions for biopsy or surgery, the latter could distinguish malignant lesions from benign ones. In fact, repeat biopsies are usually performed in patients with FUO, as was the case in this study. There-

PET/CT in FUO Resulting from Lymphoma fore, the combination could decrease the false negative rate of biopsies and increase the diagnostic rate of FUO patients with lymphoma.

This study also showed that PET/CT scan is a useful tool in diagnosing aggressive lymphomas presenting as FUO. The sensitivity and accuracy of FDG-PET/CT in diagnosing peripheral lymphomas was high in this study. In FUO patients with lymphomas, only 4 had a PET/CT result discordant with the biopsy results, 3 of them with T cell lymphoma and 1 with B cell lymphoma. This might have been caused by the fact that several subtypes of $\mathrm{T}$ cell lymphomas have very distinct biological behaviors, which may affect the FDG uptake and, therefore, vary the PET/ CT results. Given the fact that some low-grade nonHodgkin lymphomas may have low or absent FDG uptake [12], all the patients with lymphomas in this study had B symptoms (fever), suggesting advanced stage lymphomas which might have contributed to the high positive rate of FDG [13]. Fever is a common clinical manifestation of lymphoma, and it is a main syndrome - and even the only syndrome - in about $16-30 \%$ patients with malignant lymphomas [14].

Although data regarding the role of PET/CT in T cell lymphomas are limited [15], our study indicated that $\mathrm{PET} / \mathrm{CT}$ showed good performance in diagnosing $\mathrm{T}$ cell lymphomas. In the present study, 20 patients had PTCLs with an advanced stage, and $85 \%$ of them were correctly diagnosed by PET/CT. The data of our study was consistent with the National Comprehensive Cancer Network (NCCN) clinical practice guidelines (version 1, 2012), which have added PET/CT as an essential tool in the diagnosis of PTCL [16]. T cell lymphomas have a highly aggressive process, and timely diagnosis and precise staging play a significant role in the management of this subtype. Therefore, application of PET/CT in T cell lymphoma diagnosis is necessary and promising. In our study, the diagnosis of $\mathrm{T}$ cell lymphoma was confirmed by BMB in 1 patient with a normal FDG uptake. Moulin-Romsee et al. [17] reported that routine $\mathrm{BMB}$ might be unnecessary when PET/CT is negative. Several previous studies suggest that $\mathrm{BMB}$ should be considered even in FDG-negative cases $[18,19]$. Our data appeared to support the idea that $\mathrm{BMB}$ is necessary even in cases with normal FDG uptake.

In this study, $89 \%$ of the patients were referred from local hospitals, and therefore they did not represent the whole population of patients with FUO, which might have contributed to the low sensitivity rate. In addition, a variety of laboratory tests and clinical therapies had been performed in these patients before PET/CT scanning, which might have influenced the uptake of FDG in some conditions. 


\section{Conclusion}

Our study showed that PET/CT scanning was a valuable diagnostic tool for patients with FUO due to lymphoma. However, to exactly confirm the diagnostic value of PET/CT in patients with FUO resulting from lymphoma, more multicenter prospective studies with a larger number of cases are needed.

\section{Acknowledgements}

We thank Lo-Han Yuan for carefully reading our manuscript. This work was supported by the Chinese High Tech Research and Development (863) Program (No. 2011AA020104), and the National Natural Science Foundation of China (No. 30972592).

\section{Disclosure Statement} terest.

The authors declare that they have no potential conflict of in-

\section{References}

1 Petersdorf RG: Fever of unknown origin: an old friend revisited. Arch Intern Med 1992; 152:21-22.

2 Cunha BA, Mohan S, Parchuri S: Fever of unknown origin: chronic lymphatic leukemia versus lymphoma (Richter's transformation). Heart Lung 2005;34:437-441.

3 Roca Campana V, Rodriguez Silva H: Malignant lymphomas presenting as fever of unknown origin. An Med Interna 2007;24:531534.

4 Ansell SM, Armitage JO: Positron emission tomographic scans in lymphoma: convention and controversy. Mayo Clin Proc 2012;87: 571-580.

5 Hutchings M, Specht L: PET/CT in the management of haematological malignancies. Eur J Haematol 2008;80:369-380.

6 Bleeker-Rovers CP, de Kleijn EM, Corstens $\mathrm{FH}$, et al: Clinical value of FDG PET in patients with fever of unknown origin and patients suspected of focal infection or inflammation. Eur J Nucl Med Mol Imaging 2004; 31:29-37.

-7 Jaruskova M, Belohlavek O: Role of FDG-PET and PET/CT in the diagnosis of prolonged febrile states. Eur J Nucl Med Mol Imaging 2006;33:913-918.
8 Ferda J, Ferdova E, Zahlava J, et al: Fever of unknown origin: a value of ${ }^{18} \mathrm{~F}$-FDG-PET/CT with integrated full diagnostic isotropic CT imaging. Eur J Radiol 2010;73:518-525.

-9 Tokmak H, Ergonul O, Demirkol O, et al: Diagnostic contribution of ${ }^{18} \mathrm{~F}$-FDG-PET/CT in fever of unknown origin. Int J Infect Dis 2014; 19:53-58.

10 Petersdorf RG, Beeson PB: Fever of unexplained origin: report on 100 cases. Medicine (Baltimore) 1961;40:1-30.

11 Swerdlow SH, Campo E, Harris NL: WHO Classification of Tumours of Haematopoietic and Lymphoid Tissues, ed. 4. Lyon, International Agency for Research on Cancer, 2008, vol 2, pp 31-349.

12 Carr R, Barrington SF, Madan B, et al: Detection of lymphoma in bone marrow by wholebody positron emission tomography. Blood 1998;91:3340-3346.

13 Vassilakopoulos TP, Angelopoulou MK, Constantinou N, et al: Development and validation of a clinical prediction rule for bone marrow involvement in patients with Hodgkin lymphoma. Blood 2005;105:1875-1880.

$14 \mathrm{Xu}$ Y, Jin M: Diagnosis of lymphomas in fever of unknown origin cases (in Chinese). Chin J Clin Med 2009;16:640-642.
15 Feeney J, Horwitz S, Gönen M, et al: Characterization of T-cell lymphomas by FDG PET/ CT. AJR Am J Roentgenol 2010;195:333-340. 16 National Comprehensive Cancer Network: Clinical practice guidelines, 2012: NCCN guidelines for treatment of cancer by site. http://www.nccn.org/professionals/physician_gls/f_guidelines.asp\#site (accessed August 10, 2013).

17 Moulin-Romsee G, Hindie E, Cuenca X, et al: ${ }^{18} \mathrm{~F}-\mathrm{FDG}$ PET/CT bone/bone marrow findings in Hodgkin's lymphoma may circumvent the use of bone marrow trephine biopsy at diagnosis staging. Eur J Nucl Med Mol Imaging 2010;37:1095-1105.

18 Chen YK, Yeh CL, Tsui CC, et al: F-18 FDG PET for evaluation of bone marrow involvement in non-Hodgkin lymphoma: a metaanalysis. Clin Nucl Med 2011;36:553-559.

19 Schaefer NG, Strobel K, Taverna C, et al: Bone involvement in patients with lymphoma: the role of FDG-PET/CT. Eur J Nucl Med Mol Imaging 2007;34:60-67. 